\title{
Reduction of Optical Bleaching in Phosphorus doped Ge layer on $\mathrm{Si}$
}

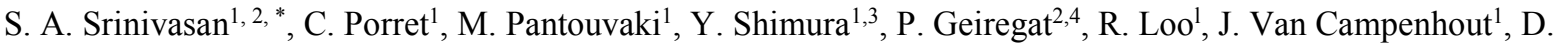 \\ Van Thourhout ${ }^{2}$ \\ ${ }^{1}$ Imec, Kapeldreef 75, Heverlee B-3001, Belgium \\ ${ }^{2}$ Photonics Research Group, Dept. of Information Technology, Ghent University - imec \& Center for Nano- and Biophotonics, Ghent \\ University, Technologiepark-Zwijnaarde 15 iGent, 9052 Ghent, Belgium \\ ${ }^{3}$ Current address: Shizuoka University, Hamamatsu 432-8001, Japan \\ ${ }^{4}$ Physics and Chemistry of Nanostructures, Ghent University, 9000 Ghent, Belgium \\ *Author e-mail address: ashwyn.srinivasan@imec.be
}

\begin{abstract}
Optical bleaching is studied on undoped and highly doped Ge layer on Si using Transient Absorption Spectroscopy. Upon optical pumping, doped Ge showed a reduction in optical bleaching as compared to undoped Ge due to the homogeneous broadening effect in doped Ge.
\end{abstract}

Index Terms — Laser; Homogeneous broadening; Germanium.

\section{INTRODUCTION}

$\mathrm{S}_{\mathrm{i}}^{\mathrm{i}}$ ilicon photonics $(\mathrm{SiPh})$ is widely considered for short-reach optical interconnect applications where their electrical counterparts face the bandwidth-power density bottleneck [1]. An important element of any SiPh based optical circuit is the light source. Due to its lattice constant and the short band gap, Germanium (Ge) is considered as a candidate for the monolithic integration of the light source in silicon photonics [2]. Ge is intrinsically an indirect band gap material with the indirect $\mathrm{L}$ conduction valley located $136 \mathrm{meV}$ below the direct $\Gamma$ conduction valley, but it can become a pseudo direct band gap material when doped with Phosphorus (P) atoms [2]. In this work, we present a comparative study of the impact of Phosphorus doping on optical bleaching (OB) using transient absorption spectroscopy (TAS). This experimental method and analysis enable benchmarking the performance of Ge laser.

\section{SAMPLE AND EXPERIMENT DETAILS}

The sample set under study in this work consists of an undoped and a highly doped Ge layer grown on Si substrates. A conventional $1 \mu \mathrm{m}$ thick Ge buffer layers grown on $300 \mathrm{~mm}$ Si (001) substrates and later thinned down to $0.6 \mu \mathrm{m}$ using chemical-mechanical polishing is used as the undoped Ge reference sample. The P-doped Ge layer of $0.2 \mu \mathrm{m}$ thickness were grown on a $0.2 \mu \mathrm{m}$ thick Ge buffer on $\mathrm{Si}$. They were deposited using an RPCVD process at $320^{\circ} \mathrm{C}$ with $\mathrm{Ge}_{2} \mathrm{H}_{6}$ and $\mathrm{PH}_{3}$ gases as precursors [3]. The doped Ge layers were later Rapid Thermal Annealed (RTA) at $700^{\circ} \mathrm{C}$ for $30 \mathrm{sec}$ in $\mathrm{N}_{2}$ in order to diffuse the P-atoms into the underlying undoped $\mathrm{Ge}$ buffer resulting in uniformly doped $0.4 \mu \mathrm{m}$ thick Ge layers. Apart from realizing a uniformly doped Ge layer, RTA also improves the crystallinity of the layer due to a reduction in point defects and increases the activation of dopants [4]. A summary of the sample set investigated in this work is presented in Table 1. The active Phosphorus concentration in the doped sample is $2.65 \times 10^{19} \mathrm{~cm}^{-3}$, similar to that of the electrically pumped Ge laser presented in [6].

Table 1: Summary of sample details investigated in this paper.

\begin{tabular}{|c|c|c|}
\hline Sample detail & Chem. P conc. & Active P conc. \\
\hline $0.6 \mu \mathrm{m}$ Ge buffer (reference) & - & - \\
\hline 0.4 um doped Ge & $2.8 \times 10^{19} \mathrm{~cm}^{-3}$ & $2.65 \times 10^{19} \mathrm{~cm}^{-3}$ \\
\hline
\end{tabular}

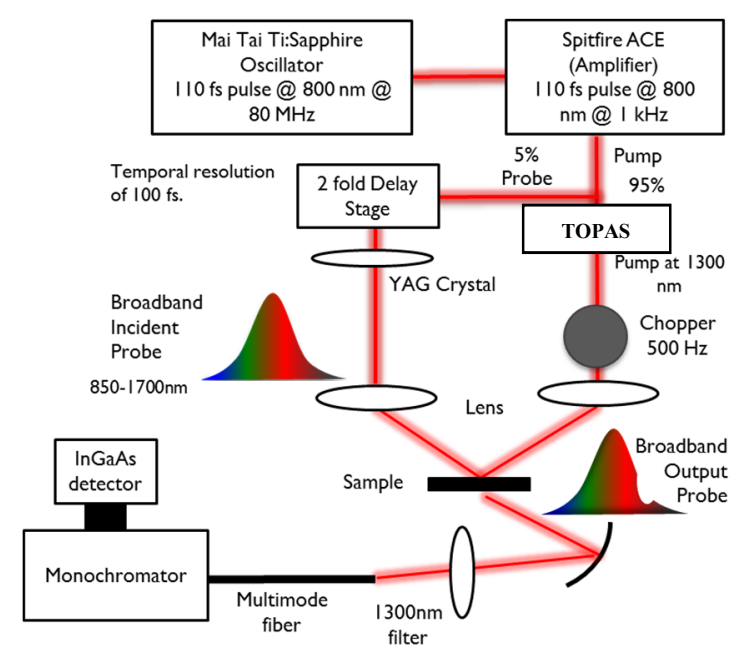

Fig. 1. Schematic of the Transient Absorption Spectroscopy experiment. The $1300 \mathrm{~nm}$ filter is used to filter out any residual pump signal transmitted through the sample.

TAS measurements are performed in this work to study optical bleaching in Ge. We measure the change in absorption of the broadband probe (1400 -1700 nm) through Ge when excited with a pump at $1300 \mathrm{~nm}$. A Mai Tai Ti:S oscillator, a Spitfire regenerative amplifier and a Travelling Wave Optical Parametric Amplifier (TOPAS) are used to generate the pump signal while a broadband probe signal is obtained from the YAG crystal. Fig. 1 shows the experimental setup. The temporal change in the absorption coefficient of the probe signal with respect to the incident pump signal is measured with the help of a two-fold delay stage. The resolution of this 
experiment is $100 \mathrm{fs}$. The measurements were performed with a pump intensity of up to $140 \mathrm{~mW} / \mathrm{cm}^{2}$.

\section{RESULTS AND DISCUSSION}

When pumping the sample under test, a high density of electrons and holes are generated in the Ge layer and then decay over time due to recombination. The temporal dynamics of this process is monitored using the broadband probe, which experiences both free-carrier absorption and OB (reduction in absorption coefficient). The net change in transmitted probe power due to the pump, expressed in terms of reduction in absorption coefficient, is reported in this work.
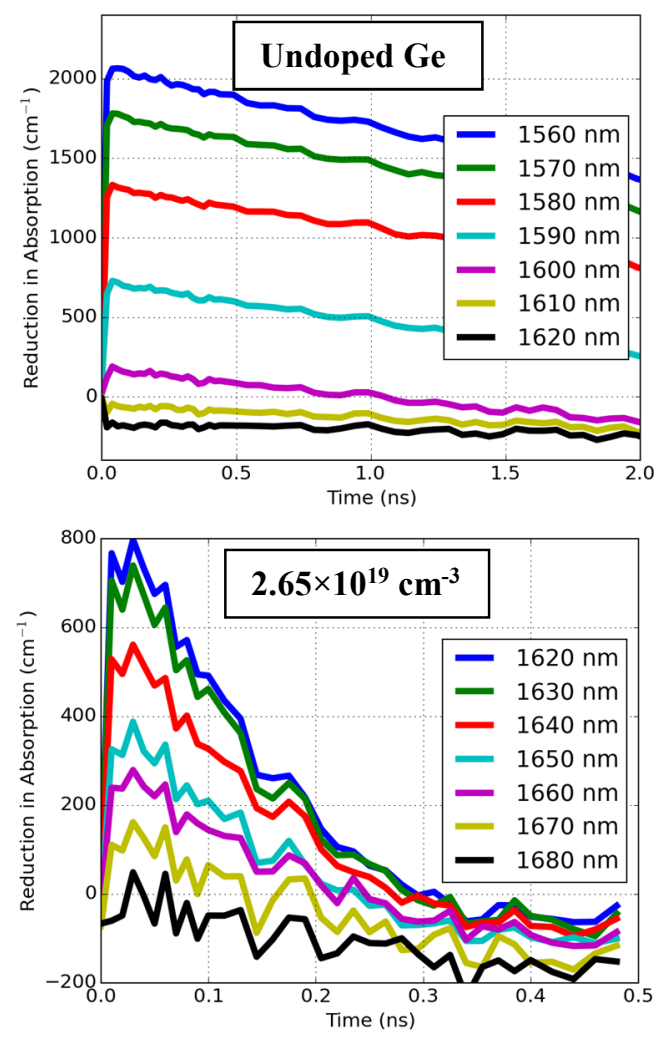

Fig. 2. Time resolved reduction in absorption co-efficient of undoped and phosphorus-doped Ge close to the band edge. The result was extracted for a pump induced excited carrier concentration of $1 \times 10^{19} \mathrm{~cm}^{-3}$.

Since the pump intensity was varied from $28-140 \mathrm{~mW} / \mathrm{cm}^{2}$, a pump induced excited carrier concentration of $0.3-2 \times 10^{19} \mathrm{~cm}^{-}$ ${ }^{3}$ was estimated using an analytical model. The measured change in absorption coefficient for an excited carrier concentration of $1 \times 10^{19} \mathrm{~cm}^{-3}$ in the doped and undoped samples is shown in Fig. 2. Since the reduction in absorption coefficient for both samples is $>0$, OB dominates the pump-induced free carrier absorption. Similar observations have been reported in $[7,8]$. However, for a fixed excited carrier concentration of $1 \times 10^{19} \mathrm{~cm}^{-3}$, optical bleaching in the doped Ge layer is suppressed as compared to that of undoped $\mathrm{Ge}$, as clearly shown in Fig. 3. This trend was observed over the entire range of excited carrier concentrations. We believe that this suppression of $\mathrm{OB}$ in the doped layer arises from carrier scattering due to dopants that leads to linewidth broadening of the recombination/gain spectrum. For uniformly doped Ge layers, homogeneous broadening of $\Gamma_{\text {ном }} \geq 45 \mathrm{meV}$ was extracted using photoluminescence spectroscopy as compared to $\Gamma_{\mathrm{HOM}}=3 \mathrm{meV}$ for undoped Ge layer [8]. These findings are consistent with the OB measurements performed in this work. Fig. 2 also shows that doping reduces the carrier lifetime from $4 \mathrm{~ns}$, for undoped $\mathrm{Ge}$, to $<0.3 \mathrm{~ns}$. This decrease in lifetime is due to the presence of dopant-induced point defects leading to non-radiative recombination and can further increase the threshold current density of the laser by a factor $>4[8,9]$.

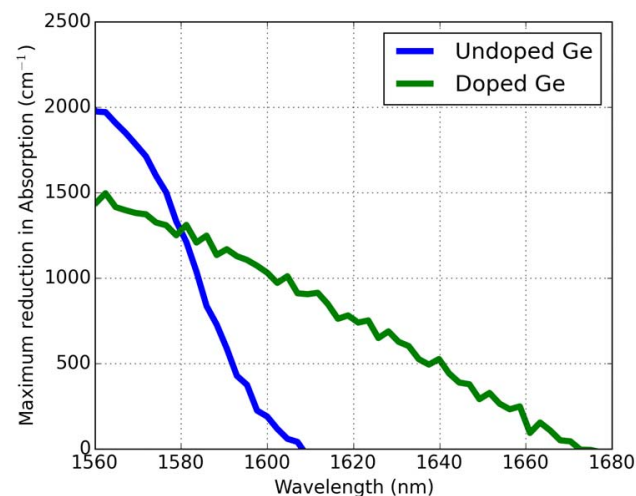

Fig. 3. Maximum optical bleaching extracted in undoped and doped $\mathrm{Ge}$ for an excited carrier concentration of $1 \times 10^{19} \mathrm{~cm}^{-3}$.

\section{CONCLUSION}

Transient Absorption Spectroscopy was performed on undoped and phosphorus-doped Ge layers on Si. Optical bleaching was observed when Ge was optically pumped. However the doped sample showed suppressed bleaching as compared to the undoped sample. We believe that this is due to dopant induced carrier scattering effects resulting in broadening of the recombination/gain spectrum. In addition, the carrier lifetime in doped Ge layers dropped by at least 1 order of magnitude due to the presence of Phosphorus induced point defects. From these findings we estimate that the threshold current density of a P-doped Ge laser is increased by a factor $>$ 4.

\section{ACKNOWLEDGEMENTS}

Y. Shimura acknowledges the Research Foundation of Flanders (FWO) for granting him a fellowship within the Pegasus Marie Curie Program. This work was supported by imec's industrial-affiliation program on Optical I/O. Air Liquide Advanced Materials is acknowledged for providing $\mathrm{Ge}_{2} \mathrm{H}_{6}$.

\section{REFERENCES}

[1] D.A.B. Miller, et al., Proc. IEEE, vol. 97, no. 7, pp. 1166-1185, Jul. 2009.

[2] J. Liu, et al., Optics Express, 15(19), 11272-77, 2007.

[3] Y. Shimura, et al., Thin Solid Films, 602, 56-69, 2016.

[4] Y. Yamamoto, et al., Material Science in Semiconductor Processing, 2016.

[5] R. Koerner, et al., Optics Express 23, 14815-14822, 2015.

[6] R. Koerner, et al., ISDTM, 2014.

[7] X. Sun, et al., ECS Transactions, 16 (10), 881-889, 2008.

[8] S. A. Srinivasan, et al., Submitted to IPC, 2017.

[9] S. A. Srinivasan, et al., Applied Physics Letters, 108(21), 211101, 2016. 